\section{Satisfacción en Estudiantes del Pregrado, en Base a su Percepción de la Calidad Docente}

\author{
Satisfaction In Undergraduate Students, Based On Their \\ Perception of Teaching Quality
}

\begin{abstract}
RESUMEN
Basado en el estudio de percepción del alumnado sobre la actividad docente de pre-grado de la Facultad de Ciencias Administrativas de la Universidad Nacional Mayor de San Marcos en el segundo semestre del 2017, en el cual se enfocaron temas como: conocimiento de la materia, asistencia y puntualidad, ética, capacidad didáctica y cumplimiento del sílabo entre otros; se plantea la presentación de un reporte personalizado al docente, donde se ubique a cada uno de sus alumnos en diferentes escenarios, los cuales han sido determinados mediante la utilización de los árboles de clasificación y regresión de la minería de datos. Con el fin de identificar patrones mediante los que el docente es valorado (competencias logradas y en proceso) y así colaborar con el mejoramiento de la calidad docente de la Facultad.
\end{abstract}

Palabras claves: Evaluación docente; minería de datos; árbol de clasificación.

\begin{abstract}
Based on the study of students' perception of the undergraduate teaching activity of the Faculty of Administrative Sciences of the National University of San Marcos in the second semester of 2017, which focused on topics such as: knowledge of the subject, attendance and punctuality, ethics, didactic capacity and compliance of the syllabus among others; the presentation of a personalized report to the teacher is proposed, where each one of his students is located in different scenarios, which have been determined through the use of classification and regression trees of data mining. In order to identify patterns through which the teacher is valued (virtues and weaknesses) and thus collaborate with the improvement of the teaching quality of the Faculty. Keywords: Teacher evaluation; data mining; classification tree.
\end{abstract}

Aurora del Carmen Molina Napurí $^{1}$

aurora.molina.n@gmail.com

Carlos Barrios Napurí ${ }^{2}$ consultorabarrios@hotmail.com

Universidad Nacional Mayor de San Marcos, Facultad de Ciencias Administrativas

\footnotetext{
1 Estadística. Egresada de la Maestría en Bioestadística. Gerente del departamento de Estadística en Stand Up Consulting. 2 Sociólogo. Egresado de la Maestría en Gestión Económica Empresarial, de la Maestría en Gestión Pública y con estudios de Doctorado en Medio Ambiente y Desarrollo Sostenible. Director de la Revista virtual indexada DELOS - Desarrollo Local Sostenible.
}

(C) Los autores. Este artículo es publicado por Gestión en el Tercer Milenio de la Facultad de Ciencias Administrativas de la Universidad Nacional Mayor de San Marcos. Este es un artículo de acceso abierto, distribuido bajo los términos de la licencia Creative Commons Atribucion - No Comercia_Compartir Igual 4.0 Internacional. (http://creativecommons.org/licenses/by-nc-sa/4.0/) que permite el uso no comercial, distribución y reproducción en cualquier medio, siempre que la obra original sea debidamente citada. 


\section{INTRODUCCIÓN}

\section{Panorama del estudio}

El Plan Estratégico Institucional 2012-2021 de la Universidad Nacional Mayor de San Marcos (UNMSM), en el Eje Estratégico 1, Educación de Calidad e Internacionalización, incluye la estrategia de garantizar la calidad y cantidad de docentes con el perfil adecuado para el desarrollo de los procesos de enseñanza-aprendizaje, investigación, tutoría, extensión universitaria y proyección social.

El presente artículo se enmarca en este Eje Estratégico y es una extensión del "Estudio de percepción del alumnado sobre la actividad docente de pre-grado, en la Facultad de Ciencias Administrativas de la Universidad Nacional Mayor de San Marcos, correspondiente al semestre académico 2017-II". Los informes del estudio para la Facultad y para cada uno de los docentes, se prepararon aplicando la estadística descriptiva. Adicionalmente, el decano de la Facultad de Ciencias Administrativas (FCA), en busca de la mejora continua de la calidad de la enseñanza aprendizaje y en la perspectiva de una óptima acreditación, invitó a continuar procesando la información acopiada a fin de explorar su mayor aprovechamiento, haciendo uso de la estadística inferencial y de otras herramientas de análisis.

\section{Objetivo}

Determinar si es viable disponer de sugerencias personalizadas para el desarrollo de capacidades de cada docente, donde se les sugiera los atributos a mejorar para alcanzar una mejor percepción entre sus estudiantes y alcanzar o mantener una evaluación satisfactoria.

\section{Referencias teóricas}

Dado que es necesario determinar los atributos de la calidad docente para alcanzar una mejor satisfacción de los estudiantes, se considera pertinente la utilización de la técnica estadística de árboles de clasificación en minería de datos, en la variante de aplicación del algoritmo CART.

La minería de datos ("Data Mining" en inglés) o exploración de datos, es el proceso de analizar y resumir grandes volúmenes de datos desde distintas perspectivas. Se aplica para en- contrar patrones y relaciones en grandes conjuntos de información, formulando algoritmos, entendidos como conjuntos de reglas validadas para resolver un problema de información, mediante una serie de pasos concretos. Las técnicas que utiliza la minería con mayor frecuencia provienen de la inteligencia artificial y de la estadística. Entre estas técnicas están los árboles de clasificación.

Los algoritmos de árbol de clasificación consisten en organizar los datos en alternativas que se agrupan formando ramas de influencia, después de una clasificación inicial. En nuestro caso, empieza con la pregunta de sí o no "recomendaría a un compañero para que lleve el curso con el docente". De ahí salen las dos ramas divergentes del árbol, y cada elección posterior tiene sus propias ramas divergentes que llevan a diversos escenarios.

El algoritmo CRT o CART (de sus siglas en inglés, Classification And Regression Trees) es un método no-paramétrico de segmentación binaria donde el árbol es construido dividiendo repetidamente los datos. El nodo inicial es llamado nodo raíz o grupo madre y se divide en dos grupos hijos o nodos, mutuamente excluyentes, luego el procedimiento de partición es aplicado a cada grupo hijo por separado (Deconinck, Zhang, Coomans, \& Heyden, 2006).

\section{Antecedentes por variable}

Previamente, tanto en el segundo semestre del 2016 como en el primer semestre del 2017, se realizaron estudios similares en esta Facultad, que permitieron validar los instrumentos de gestión, recojo y procesamiento de la información y afinar la metodología.

- En el estudio del segundo semestre del 2016, se validó todo el cuestionario (Molina \& Barrios, 2016). Se adicionó la variable endógena Satisfacción, cuyo indicador también fue validado para siguientes estudios; pero, en su procesamiento surgió una observación. Esta variable tuvo opciones de respuesta en escala Likert; por lo que, al ser ordinal, correspondía procesarla como regresión logística. La dificultad fue que, al procesar esta regresión logística en escala Likert se genera una amplitud de detalles de escaso 
Tabla 1.

Cobertura Estudio de percepción de la actividad docente FCA, pre-grado 2017-II

\begin{tabular}{|c|c|c|c|c|}
\hline & & Escuela Profesion & & \\
\hline & Administración & Administración de Turismo & $\begin{array}{l}\text { Administración de Negocios } \\
\text { Internacionales }\end{array}$ & $\begin{array}{l}\text { Facultad de Ciencias } \\
\text { Administrativas }\end{array}$ \\
\hline Cursos & 38 & 34 & 39 & 111 \\
\hline Docentes & 86 & 59 & 60 & 175 \\
\hline Encuestas & 3,471 & 2,028 & 2,298 & 7,797 \\
\hline
\end{tabular}

Fuente: Molina \& Barrios (2017)

Elaboración: Propia

aporte interpretativo, por lo cual este método se descartó.

- Como alternativa, se intentó el procesamiento en base a la obtención de promedios por sección o docente, aplicando la regresión múltiple. La desventaja de esta otra opción metodológica fue que aportaba resultados con sustentación débil, dado que se perdía información, al basar el análisis ya no en las respuestas de cada alumno sino en los promedios de respuestas obtenidos por cada sección o docente.

- En el estudio del primer semestre del 2017 se tuvo algunos cambios (Molina \& Barrios, 2017a). El principal fue que el indicador de la variable Satisfacción pasó a tener una escala dicotómica. Ello significó que el procesamiento regresó de la estadística paramétrica a la no paramétrica, sustituyendo la regresión múltiple por la regresión logística.

- Si bien es cierto que existen varios métodos estadísticos que caracterizan a una determinada población, como son las ecuaciones estructurales y los análisis multinivel, estos métodos se descartaron porque, además de ser complejos, resultan innecesarios para nuestros fines.

\section{MÉTODOS}

\section{Universo y muestra}

La FCA de la UNMSM, en el segundo semestre del 2017, solicitó a los alumnos a participar en un Estudio de Percepción sobre la Actividad Do- cente. El objetivo del estudio fue disponer de información de las percepciones de los alumnos de las tres Escuelas Académico Profesionales (Escuela Profesional) del pregrado de la FCA de la UNMSM, para la evaluación a los docentes. La cobertura alcanzada fue la siguiente (Ver Tabla 1).

\section{Variables}

El cuestionario aplicado fue elaborado por expertos de la UNMSM y aprobado con las Resoluciones Rectorales: № 04935-R-16 (Reglamento de evaluación para la ratificación docente de la UNMSM) y № 04937-R-16 (Reglamento de evaluación para la promoción docente de la UNMSM). La información recogida corresponde a un conjunto de indicadores cuantificables referidos a la percepción que los alumnos tienen de sus docentes (Ver Tabla 2).

Se adicionó la variable Satisfacción de los estudiantes con su docente.

\section{Procesamiento}

Los resultados de la encuesta se presentaron como la evaluación promedio general de la Facultad y de cada Escuela Profesional, relevando la percepción de los docentes a nivel consolidado y por preguntas del cuestionario.

Para dar valor agregado a los datos obtenidos, se necesitó conocer si es viable proveer de información personalizada a cada docente, con pautas orientadoras obtenidas de los resultados de las encuestas de evaluación, donde se les sugiera los atributos a mantener o mejorar para alcanzar una mejor percepción entre sus estudiantes y así lograr o consolidar la evaluación satisfactoria. Esto fue posible alcanzar mediante el algoritmo CART. 
Tabla 2.

Variables del estudio

\begin{tabular}{ll} 
Áreas temáticas & Variables \\
A. Conocimiento de la materia & 1. Demuestra dominio de la materia. \\
& 2. Desarrolla los contenidos con amplitud y profundidad. \\
& 3. Sus exposiciones son entendibles y comprensibles. \\
& 4. Demuestra solvencia frente a las preguntas planteadas por los alumnos sobre el tema. \\
\hline B. Asistencia y puntualidad & 5. Asiste a clases de acuerdo a lo programado en el sílabo. \\
& 6. Cumple puntualmente con el horario establecido. \\
\hline C. Ética & 7. Demuestra valores éticos como docente del curso. \\
\hline D. Capacidad didáctica & 8. Utiliza estrategias didácticas. \\
& 9. Promueve el aprendizaje activo y autónomo. \\
& 10. La evaluación del aprendizaje del alumno es objetiva de acuerdo a lo establecido. \\
\hline E. Cumplimiento del sílabo & 11. Cumple la programación de acuerdo a lo establecido en el sílabo. \\
\hline F. Otros aspectos & $\begin{array}{l}\text { 12. Hace uso de herramientas TiCs (presentaciones PowerPoint, video conferencias, aula virtual, etc.) } \\
\text { 13. Promueve el uso de otros idiomas. }\end{array}$ \\
14. Hace uso de información motivadora. \\
15. Desarrolla actividades de: Tutoría, asesoría, investigación formativa, responsabilidad social univer- \\
sitaria y extensión.
\end{tabular}

Fuente: Molina \& Barrios (2017)

\section{RESULTADOS}

\section{Resultados por variables}

Mediante la aplicación del algoritmo CART, se obtiene el árbol de clasificación (Figura 01), donde el color rojo (barra izquierda) representa los alumnos que no recomendarían a su profesor y el color azul (barra derecha) a los que no perciben la existencia de problemas y si lo recomendarían.

El parámetro del "Desarrollo de contenidos" para la "Recomendación", donde el punto de corte es la categoría de "regular". Esto es, no todas las personas que califican al docente como regular o menos en su "Desarrollo de contenidos", van a NO recomendarlo. De igual forma, no todos los que lo clasifiquen por encima de regular, van a recomendarlo. Es por ello que se tiene en cuenta otros factores, que aparecen en los niveles inferiores del árbol, para obtener la óptima clasificación.

Las variables que, según el alumnado de la Facultad, son determinantes para que estos recomienden o no a sus docentes son, sin ningún orden específico:

- Dominio de la materia

- Desarrolla contenidos a profundidad
- Exposiciones comprensibles

- Utiliza estrategias didácticas

- Evaluación objetiva

- Cumple la programación del sílabo

- Uso de TIC

- Información motivadora

- Desarrolla actividades

\section{Resultados por valores de las variables}

Las variables en el estudio son de carácter categórico, en escala Likert de 5 categorías (deficiente, regular, aceptable, bueno, excelente). Entre el conjunto de variables determinantes para la recomendación o no de los alumnos a los docentes, la principal es el "Desarrollo de contenidos". En esta variable si los resultados arrojan un valor igual o menor que regular, el $72.7 \%$ no va a recomendar al docente, mientras que el 27.3 si lo recomendaría. Pero, si la respuesta es más que regular, el $89.9 \%$ si lo recomendarían y el $10.1 \%$ no lo haría.

De esta manera, "Desarrollo de contenidos" es la variable principal que va a determinar si el alumno recomienda o no a su docente. Mediante el árbol de clasificación se obtiene 


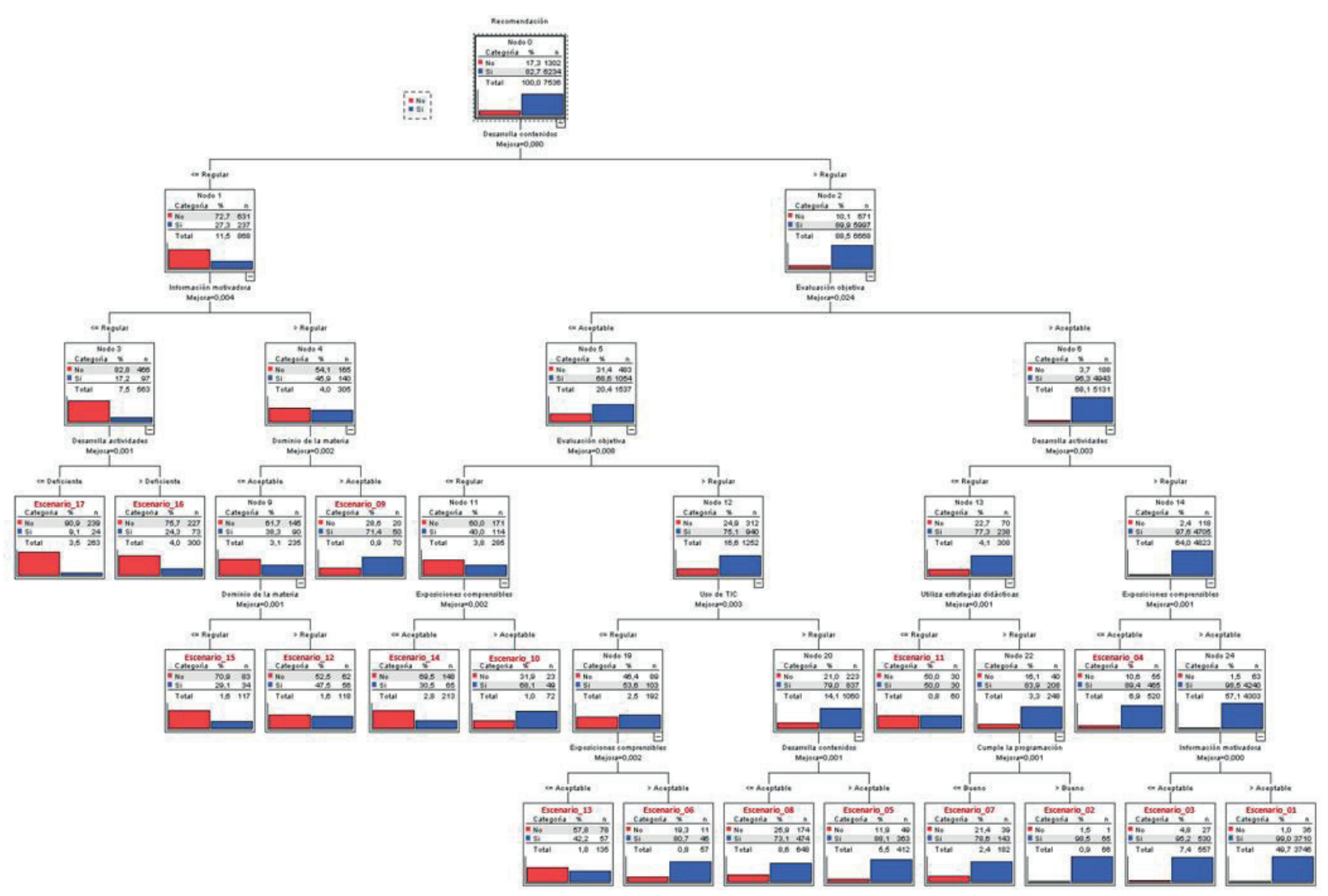

Figura 1. Árbol de clasificación sobre la recomendación del docente 2017-II de la FCA de la UNMSM.

así a grupos de alumnos con características similares y la identificación de las principales variables que los agrupan de acuerdo a su percepción. Continuando con el análisis, en el caso que el "Desarrollo de contenidos" tenga una respuesta mayor a regular, la siguiente variable en importancia es la "Evaluación objetiva". En esta variable, si la respuesta es menor o igual que aceptable, el riesgo de no ser recomendado se incrementa y si adicionalmente la respuesta está por debajo o igual a regular, es un hecho que el $60 \%$ de ellos no lo recomiende. Y así sucesivamente, en todas las ramas del árbol de clasificación.

\section{Resultados por grupos de variables (es- cenarios)}

Todo el análisis anterior hace referencia a la percepción del alumnado dentro de la FCA de la UNMSM en el semestre académico 2017-II, donde se han identificado 17 escenarios, tal como se percibe en la figura 1 , donde el más alto nivel de aceptación se observa en escenario_1 y el menor en el escenario_17. Con los resultados observados en el árbol de clasificación se tiene que, probablemente en una siguiente evaluación, si no se presentan cambios sustanciales en el desempeño docente, el $17.3 \%$ del alumnado no recomiende a sus docentes, tal como se observa en el nodo principal del árbol de clasificación.

De esta manera, parte del reporte personalizado al docente incluye distribuir a sus alumnos dentro de los diferentes escenarios e identificar patrones que los caractericen. Estos patrones determinados por los tipos de respuestas a las variables principales, permitirían sugerir al docente cuáles son los puntos cruciales, sobre los cuales deberían desarrollar sus capacidades y así obtener mejoras tanto en la percepción de su alumnado como en la calificación resultante de su evaluación.

Los 17 escenarios mencionados se muestran en la tabla 3. 
Tabla 3.

Especificación de escenarios

\begin{tabular}{|c|c|c|c|c|c|c|}
\hline Escenario & Variable & Deficiente & Regular & $\begin{array}{c}\text { Escala } \\
\text { Aceptable }\end{array}$ & Bueno & Excelente \\
\hline \multirow[t]{5}{*}{01} & Desarrolla contenidos & & & $\mathrm{x}$ & $\mathrm{x}$ & $\mathrm{x}$ \\
\hline & Evaluación objetiva & & & & $\mathrm{x}$ & $\mathrm{x}$ \\
\hline & Desarrolla actividades & & & $\mathrm{x}$ & $\mathrm{x}$ & $\mathrm{x}$ \\
\hline & Exposiciones comprensibles & & & & $\mathrm{x}$ & $\mathrm{x}$ \\
\hline & Información motivadora & & & & $\mathrm{x}$ & $\mathrm{x}$ \\
\hline \multirow[t]{5}{*}{02} & Desarrolla contenidos & & & $\mathrm{x}$ & $\mathrm{x}$ & $\mathrm{x}$ \\
\hline & Evaluación objetiva & & & & $\mathrm{x}$ & $\mathrm{x}$ \\
\hline & Desarrolla actividades & & & $\mathrm{x}$ & $\mathrm{x}$ & $\mathrm{x}$ \\
\hline & Utiliza estrategias didácticas & & & $\mathrm{x}$ & $\mathrm{x}$ & $\mathrm{x}$ \\
\hline & Cumple la programación del sílabo & & & & & $\mathrm{x}$ \\
\hline \multirow[t]{5}{*}{03} & Desarrolla contenidos & & & $\mathrm{x}$ & $\mathrm{x}$ & $\mathrm{x}$ \\
\hline & Evaluación objetiva & & & & $\mathrm{x}$ & $\mathrm{x}$ \\
\hline & Desarrolla actividades & & & $\mathrm{x}$ & $\mathrm{x}$ & $\mathrm{x}$ \\
\hline & Exposiciones comprensibles & & & & $\mathrm{x}$ & $\mathrm{x}$ \\
\hline & Información motivadora & $\mathrm{x}$ & $\mathrm{x}$ & $\mathrm{x}$ & & \\
\hline \multirow[t]{4}{*}{04} & Desarrolla contenidos & & & $\mathrm{x}$ & $\mathrm{x}$ & $\mathrm{x}$ \\
\hline & Evaluación objetiva & & & & $\mathrm{x}$ & $\mathrm{x}$ \\
\hline & Desarrolla actividades & & & $\mathrm{x}$ & $\mathrm{x}$ & $\mathrm{x}$ \\
\hline & Exposiciones comprensibles & $\mathrm{x}$ & $\mathrm{x}$ & $\mathrm{x}$ & & \\
\hline \multirow[t]{3}{*}{05} & Desarrolla contenidos & & & & $\mathrm{x}$ & $\mathrm{x}$ \\
\hline & Evaluación objetiva & & & $\mathrm{x}$ & & \\
\hline & Uso de TIC & & & $\mathrm{x}$ & $\mathrm{x}$ & $\mathrm{x}$ \\
\hline \multirow[t]{4}{*}{06} & Desarrolla contenidos & & & $\mathrm{x}$ & $\mathrm{x}$ & $\mathrm{x}$ \\
\hline & Evaluación objetiva & & & $\mathrm{x}$ & & \\
\hline & Uso de TIC & $\mathrm{x}$ & $\mathrm{x}$ & & & \\
\hline & Exposiciones comprensibles & & & & $\mathrm{x}$ & $\mathrm{x}$ \\
\hline \multirow[t]{5}{*}{07} & Desarrolla contenidos & & & $\mathrm{x}$ & $\mathrm{x}$ & $\mathrm{x}$ \\
\hline & Evaluación objetiva & & & & $\mathrm{x}$ & $\mathrm{x}$ \\
\hline & Desarrolla actividades & & & $\mathrm{x}$ & $\mathrm{x}$ & $\mathrm{x}$ \\
\hline & Utiliza estrategias didácticas & & & $\mathrm{x}$ & $\mathrm{x}$ & $\mathrm{x}$ \\
\hline & Cumple la programación del sílabo & $\mathrm{x}$ & $\mathrm{x}$ & $\mathrm{x}$ & $\mathrm{x}$ & \\
\hline \multirow[t]{3}{*}{08} & Desarrolla contenidos & & & $\mathrm{x}$ & & \\
\hline & Evaluación objetiva & & & $\mathrm{x}$ & & \\
\hline & Uso de TIC & & & $\mathrm{x}$ & $\mathrm{x}$ & $\mathrm{x}$ \\
\hline \multirow[t]{3}{*}{09} & Desarrolla contenidos & $\mathrm{x}$ & $\mathrm{x}$ & & & \\
\hline & Información motivadora & & & $\mathrm{x}$ & $\mathrm{x}$ & $\mathrm{x}$ \\
\hline & Dominio de la materia & & & & $\mathrm{x}$ & $\mathrm{x}$ \\
\hline \multirow[t]{3}{*}{10} & Desarrolla contenidos & & & $\mathrm{x}$ & $\mathrm{x}$ & $\mathrm{x}$ \\
\hline & Evaluación objetiva & $\mathrm{x}$ & $\mathrm{x}$ & & & \\
\hline & Exposiciones comprensibles & & & & $\mathrm{x}$ & $\mathrm{x}$ \\
\hline \multirow[t]{4}{*}{11} & Desarrolla contenidos & & & $\mathrm{x}$ & $\mathrm{x}$ & $\mathrm{x}$ \\
\hline & Evaluación objetiva & & & & $\mathrm{x}$ & $\mathrm{x}$ \\
\hline & Desarrolla actividades & & & $\mathrm{x}$ & $\mathrm{x}$ & $\mathrm{x}$ \\
\hline & Utiliza estrategias didácticas & $\mathrm{x}$ & $\mathrm{x}$ & & & \\
\hline \multirow[t]{3}{*}{12} & Desarrolla contenidos & $\mathrm{x}$ & $\mathrm{x}$ & & & \\
\hline & Información motivadora & & & $\mathrm{x}$ & $\mathrm{x}$ & $\mathrm{x}$ \\
\hline & Dominio de la materia & & & $\mathrm{x}$ & & \\
\hline \multirow[t]{4}{*}{13} & Desarrolla contenidos & & & $\mathrm{x}$ & $\mathrm{x}$ & $\mathrm{x}$ \\
\hline & Evaluación objetiva & & & $\mathrm{x}$ & & \\
\hline & Uso de TIC & $\mathrm{x}$ & $\mathrm{x}$ & & & \\
\hline & Exposiciones comprensibles & $\mathrm{x}$ & $\mathrm{x}$ & $\mathrm{x}$ & & \\
\hline \multirow[t]{3}{*}{14} & Desarrolla contenidos & & & $\mathrm{x}$ & $\mathrm{x}$ & $\mathrm{x}$ \\
\hline & Evaluación objetiva & $\mathrm{x}$ & $\mathrm{x}$ & & & \\
\hline & Exposiciones comprensibles & $\mathrm{x}$ & $\mathrm{x}$ & $\mathrm{x}$ & & \\
\hline 15 & Desarrolla contenidos & $\mathrm{x}$ & $\mathrm{x}$ & & & \\
\hline & Información motivadora & & & $\mathrm{x}$ & $\mathrm{x}$ & $\mathrm{x}$ \\
\hline & Dominio de la materia & $\mathrm{x}$ & $\mathrm{x}$ & & & \\
\hline 16 & Desarrolla contenidos & $\mathrm{x}$ & $\mathrm{x}$ & & & \\
\hline & Información motivadora & $\mathrm{x}$ & $\mathrm{x}$ & & & \\
\hline & Desarrolla actividades & & $\mathrm{x}$ & $\mathrm{x}$ & $\mathrm{x}$ & $\mathrm{x}$ \\
\hline 17 & Desarrolla contenidos & $\mathrm{x}$ & $\mathrm{x}$ & & & \\
\hline & Información motivadora & $\mathrm{x}$ & $\mathrm{x}$ & & & \\
\hline & Desarrolla actividades & $\mathrm{x}$ & & & & \\
\hline
\end{tabular}

Elaboración: Propia 


\section{DISCUSIÓN}

Contrastando los escenarios identificados con el objetivo de determinar si es viable disponer de recomendaciones personalizadas para el desarrollo de capacidades de cada docente, se presenta a continuación un par de casos reales de la FCA. En ellos, uno de los docentes está recomendado por sus alumnos y el otro no.

\section{Caso docente " $A$ ":}

Los resultados de este docente indican que el $80 \%$ de los alumnos lo recomienda. Para este caso las sugerencias personalizadas se orientan a fortalecer la satisfacción de los alumnos.

En el análisis estadístico, mediante el árbol de clasificación planteado y ubicando a todos sus alumnos dentro de los diferentes escenarios, se observa que el $20 \%$ que no lo recomienda, se localiza en el escenario_11.

Con el fin de brindar al docente sugerencias personalizadas para consolidar o mejorar su calificación, se le brindará los siguientes resultados:

Estimado docente "A":

Usted se encuentra entre el $83 \%$ de docentes de pregrado de la FCA-UNMSM que, en el semestre académico 2017-II, al ser evaluados por sus estudiantes, tuvo mayoritariamente una recomendación favorable como docente.

Felicitaciones.

Es importante que esta satisfacción mayoritaria se mantenga y consolide en los siguientes semestres. Los resultados de su evaluación muestran que el $20 \%$ de sus alumnos se enmarca en el escenario_11, donde la probabilidad de que sea recomendado es del $50 \%$. El solo hecho de modificar la percepción de este $20 \%$ de alumnos en la variable "Estrategias didácticas", a aceptable como mínimo, incrementa su probabilidad de recomendación hasta un $29 \%$.

En otras palabras (en el supuesto que el docente tuviera 100 alumnos), si tan solo 20 de sus alumnos cambiaran su opinión de malo o regular a aceptable en la pregunta de la utilización de estrategias didácticas, usted mejoraría notablemente.

\section{Caso docente "B":}

Los resultados de este docente indican que solo el $52 \%$ de sus alumnos lo recomiendan, por lo que este docente muestra síntomas de no aceptación por casi la mitad del alumnado. Sin embargo, tiene la posibilidad de mejorar, pese a que un $48 \%$ de su alumnado no lo califica satisfactoriamente.

Mediante el análisis estadístico y haciendo uso de la técnica del árbol de clasificación planteado, se realiza una ubicación de todos los alumnos del docente, determinando así cuál o cuáles son los escenarios donde estos principalmente se concentran. Así, en el escenario_15 se alberga al 27\% de su alumnado, estando el 21\% restante entre los escenarios 13 y 12.

Con el fin de mostrar al docente cuáles son sus alternativas de mejora se le presenta el siguiente reporte:

Estimado docente "B":

Lamentablemente usted no se encuentra entre del 83\% de docentes de pregrado de la FCAUNMSM que, en el semestre académico 2017-II, al ser evaluados por sus estudiantes, tuvo mayoritariamente una recomendación favorable como docente.

Esperamos tome en cuenta las siguientes sugerencias.

Debido a que el $27 \%$ de todo su alumnado tiende a caer en el escenario_15, donde probablemente no lo recomienden, es necesario saber que con fines de mejorar los resultados de su evaluación, usted tendría que:

- Esforzarse para que el desarrollo de sus contenidos llegue a ser aceptable como mínimo, que sea bueno evaluando objetivamente y que sean aceptables el desarrollo de actividades. Para así poder posicionarse en uno de los mejores niveles.

- $\quad$ Sin embargo, si la opción anterior no le es posible, su única mejor alternativa sería que sea bueno el dominio de la materia; con este resultado usted podría invertir la probabilidad de aceptación.

Si lograse cualquiera de los cambios anteriores, las probabilidades de aceptación mejorarían enormemente. 


\section{CONCLUSIONES}

Bajo los resultados enfocados para el presente artículo sobre el estudio realizado por la FCA de la UNMSM en el 2017-II, se está en la posibilidad de informar a cada uno de los docentes, de forma personalizada, no solo cuál es su situación actual sobre la percepción entre sus estudiantes sobre sus atributos docentes, sino además proporcionarle la posibilidad de identificar cuáles son sus fortalezas y debilidades, para así ayudarlo a mejorar su posición en la satisfacción estudiantil. Esto es así por la posibilidad de aplicar las técnicas estadísticas mencionadas para minería de datos en la realización de ese estudio, aportando una herramienta necesaria a la Facultad para mejorar el alcance de sus metas y desafíos.

Los resultados y análisis que se pueden obtener con la minería de datos, son muy amplios. Se recomienda inicialmente poner atención a los resultados personalizados y evaluar, según las tendencias, la posibilidad de capacitar a los docentes precisamente en los aspectos en que la mayoría de ellos requiera reforzar.

\section{AGRADECIMIENTO}

Los análisis realizados en el presente artículo hacen mención al estudio de evaluación docente de la FCA de la UNMSM en el semestre académi- co 2017-II. Estos análisis no hubieran sido posible realizarlos sin la autorización previa del uso de la base de datos, otorgada por el decano de la Facultad, Mg. Robert A. Miranda Castillo; quien, con su valioso apoyo y constante deseo de mejora en la educación universitaria, incentivó la realización de la presente investigación.

\section{REFERENCIAS BIBLIOGRÁFICAS}

Deconinck, E., Zhang, M., Coomans, D., \& Heyden, Y. (2006). Classification tree models for the prediction of blood-brain barrier passage of drugs. En Journal of Quemical Information and Modeling (Vol. 3, págs. 1410-1419).

Fernández Nogales, Á. (2004). En Investigación y Técnicas de Mercado (2 ed., págs. 154-160). Madrid, España: ESIC.

Molina, Aurora \& Barrios, Carlos (2016). "Estudio de percepción del alumnado sobre la actividad docente de la Facultad de Ciencias Administrativas de la UNMSM 2016-II" Inédito.

Molina, Aurora \& Barrios, Carlos (2017a). "Estudio de percepción del alumnado de pregrado sobre la actividad docente en la Facultad de Ciencias Administrativas de la UNMSM 2017-I Inédito.

Molina, Aurora \& Barrios, Carlos (2017b). "Estudio de percepción del alumnado sobre la actividad docente de pre-grado en la Facultad de Ciencias Administrativas de la Universidad Nacional Mayor de San Marcos, correspondiente al semestre académico 2017-II". Inédito. 